\title{
Executivos Com Maior Sofisticação Financeira São Mais Confiantes e Otimistas?
}

\section{Are More Financial Sophisticated Executives Overconfidents and Optimists?}

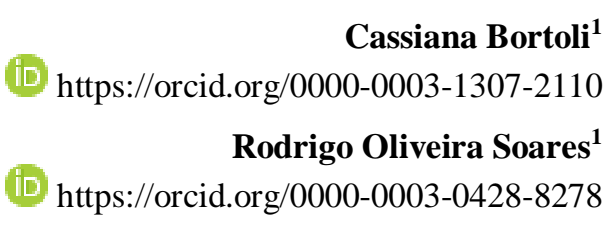

Universidade Federal do Paraná, Grupo de Pesquisa Laboratório de Finanças, Curitiba, PR, Brasil ${ }^{1}$

Artigo recebido em 25.05.2018. Última versão recebida em 06.12.2018. Aprovado em 07.12.2018. 


\title{
Resumo
}

A competitividade no mercado de trabalho de altos executivos, e busca de qualificação dos Chief Executive Officer (CEO) por parte das empresas pode levar esse profissional a superestimar suas capacidades e/ou subestimarem os riscos nos processos de tomada de decisão, aumentando a propensão de que ele apresente os vieses cognitivos excesso de confiança e otimismo (ECO). Nesse sentido, o presente trabalho teve por objetivo identificar a relação entre a Sofisticação Financeira dos CEO's e os vieses cognitivos Excesso de Confiança e Otimismo. A amostra compreendeu 179 empresas listadas na BM\&FBovespa durante os períodos de 2011 a 2015, totalizando 810 observações. A análise de componentes principais foi utilizada para a formação de três índices de sofisticação financeira: O Índice de Sofisticação Financeira Acadêmica (ISFA), o Índice de Sofisticação Financeira Profissional (ISFP), e o Índice de Sofisticação Financeira (ISF), composto pelos dois primeiros. Na análise de dados foram utilizadas regressões lineares multivariadas. Os resultados indicaram que apesar de o ISF não estar relacionado com os vieses ECO, o ISFA apresentou-se positiva e significativamente relacionado ao ECO, indicando que os CEO's que possuem formação internacional, formação financeira e experiência no ramo são propensos a apresentar os desvios cognitivos ECO.

Palavras-chave: sofisticação financeira; formação acadêmica; experiência profissional; excesso de confiança; otimismo.

\begin{abstract}
Competitiveness in top executive job market, and the companies search for Chief Executive Officer (CEO) qualification may lead that professional to overestimate his abilities and/or to underestimate the risks in decision making process, increasing his propensity to present the cognitive biases of overconfidence and optimism (ECO). Hence, the present work aimed to identify the relationship among the Financial Sophistication of CEO's and the Cognitive Biases Overconfidence and Optimism. The sample approached 179 companies listed at BM\&FBovespa during the periods from 2011 to 2015 , totaling 810 observations. We used the principal components analysis to build three financial sophistication indexes: Academic Financial Sofistication Index (ISFA), Professional Financial Sofistication Index (ISF), and Financial Sophistication Index (ISF), which is a composition of the first two. We perform Data analysis using a multivariate linear regression method, to test the relations. The results pointed that despite the ISF is not related to the ECO biases, the ISFA was positively and significantly related to the ECO, indicating that CEOs with international training, financial training and experience in the field are likely to present deviations cognitive ECO.
\end{abstract}

Keywords: financial sophistication index; academic financial sophistication index; professional financial sophistication index; overconfidence; optimism.

JEL Code: C91, D9, C43. 


\section{Introdução}

Pesquisas relativas ao comportamento humano têm revelado que as decisões do ser humano são afetadas pelo uso sistemático de um conjunto de regras simplificadoras nos seus processos de julgamento e de escolha (Barros, 2005). O autor descreve que uma das linhas de pesquisa mais férteis em finanças comportamentais, dedica-se à modelagem do comportamento financeiro com base nos agentes que apresentam algum viés cognitivo catalogado pelos psicólogos e pesquisadores comportamentais. Barros (2005) ressalta que, dentre os vieses cognitivos, o excesso de confiança e o otimismo tem conseguido explicar uma parcela significativa da distância entre as características do comportamento humano e o paradigma de racionalidade perfeita, abrangendo grande parte dos fenômenos relevantes para a área de Finanças.

Os vieses excesso de confiança e otimismo podem estar intrínsecos ao próprio processo de seleção profissional (Kausel, Culbertson, \& Madrid, 2016). Dentre os fatores considerados no momento de contratação ou eleição para cargos de elevada hierarquia, Oliva e Albuquerque (2007) apontaram o conhecimento em economia, finanças, contabilidade, mercado nacional e internacional, setores específicos de atuação da empresa, e experiências profissionais de cargos já exercidos anteriormente e que possam manter relações profissionais de interesse da empresa. Manfredi (1998) revela que o fato das empresas procurarem profissionais qualificados, motiva uma maior qualificação, em média, do que é considerado necessário para desenvolver as atividades atribuídas ao cargo, provocando o que os autores titulam por supereducação ou superqualificação.

O capital intelectual de uma empresa proporciona maior inteligência, sofisticação e integração à economia, de forma a ampliar as chances de sobrevivência frente a um mercado competitivo (Rezende, 2002). Assim, profissionais que assumem cargos da alta administração, tendem a obter elevado conhecimento e experiências profissionais relacionados à área financeira, aqui denominados Sofisticação Financeira. Cabe ressaltar que o construto de Sofisticação Financeira reúne uma série de itens já pesquisados, principalmente em estudos internacionais.

A Sofisticação Financeira é desejada pelos acionistas, devido a acreditarem que um maior conhecimento e experiência profissional relacionados à área de finanças, tornam o gestor mais racional em suas decisões. Contudo, o excesso de confiança pode impactar no processo de tomada de decisão nos seus três estágios, como descrito por Lambert, Bessière e N'Goala (2012): julgamento, avaliação e decisão, de modo a superestimar os conhecimentos, acreditar que sua a probabilidade de acertos é superior à média e ter a ilusão do controle, respectivamente. $\mathrm{O}$ julgamento depende diretamente dos conhecimentos prévios; os quais serão utilizados no processo de avaliação, tendo em vista efetuar previsões; e somente posteriormente são expressas as convicções em relação a melhor atitude a ser tomada na fase decisória.

Lambert et al. (2012) destacam a importância de abranger duas amostras distintas nos estudos relacionados à tomada de decisões, uma composta por alunos de graduação e mestrado de economia e finanças, e outra por profissionais do setor financeiro. Esse fato enaltece a suposição, de que os conhecimentos acadêmicos e experiência profissional podem estar relacionados aos desvios cognitivos excesso de confiança e otimismo. Partindo desta conjectura, explicita-se a seguinte questão de pesquisa: Qual a relação existente entre a Sofisticação Financeira dos CEO's e os vieses cognitivos Excesso de Confiança e Otimismo?

Para tanto, mensurou-se a sofisticação financeira dos CEO's mediante análise fatorial com extração por meio de análise de componentes principais, tendo em vista considerar pesos diferentes para cada um dos itens integrantes ao índice. O excesso de confiança e otimismo do CEO foi calculado a partir da divisão: compra de ações da própria empresa/venda de ações da própria empresa. Para a avaliação dessa última variável consideraram-se conceitos e raciocínios utilizados para a mensuração de tais vieses em estudos internacionais adaptados à disponibilidade de dados do contexto brasileiro. A fim de responder à indagação de pesquisa, realizaram-se testes de regressão considerando dois modelos econométricos (o primeiro considerando o Índice de Sofisticação Financeira; e o segundo considerando 
índices distintos para as dimensões Acadêmica e Profissional da sofisticação financeira). Também foram testados modelos adicionais a fim de aumentar a robustez do estudo, controlando setor e ano. Os resultados apontaram que o Índice de Sofisticação Financeira Acadêmica é capaz de explicar o excesso de confiança e otimismo, ou seja, os itens ocupantes da dimensão acadêmica: Formação Acadêmica, Formação Internacional e Experiência no Ramo possuem poder explicativo no comportamento de desvio de racionalidade observado. De outra forma, pode-se dizer ainda que, a sofisticação acadêmica do CEO tem conduzido o mesmo superestimar suas capacidades, de forma a apostarem que os reflexos de sua gestão irão incidir na elevação do preço da ação da empresa, e/ou a subestimar os benefícios da diversificação de seu risco idiossincrático, investindo na empresa em que atuam.

Nesse sentido, o presente estudo contribui com a literatura de finanças comportamentais, em especial sobre o excesso de confiança e otimismo, ao mostrar que características curriculares do CEO podem estar relacionadas a estes vieses. Espera-se no Brasil uma maior heterogeneidade de formação do CEO. Além disto, a existência de maior volatilidade no mercado, pode oportunizar a explicitação do comportamento de overconfidence através de movimentos de compra de ações. Adicionalmente, a concentração acionária no mercado brasileiro pode ser propícia ao estudo de excesso de confiança, pois se já existe concentração, a compra líquida de ações indica uma confiança ou otimismo excessivos. Dessa forma, a adaptação de uma proxy para o excesso de confiança e otimismo torna possível a captação desses vieses em um contexto nacional. Já que, no decorrer do estudo, identificou-se uma carência de estudos nacionais que abordem o excesso de confiança e otimismo, e um dos motivos pode ser a dificuldade de replicar métricas já utilizadas. A identificação da relação de características curriculares com os vieses pode contribuir no sentido de indicar uma possível proxy a ser desenvolvida para o excesso de confiança e otimismo. Outro ponto a ressaltar em termos de contribuição é em relação às políticas de contratação das empresas, ou aos mecanismos de governança a serem considerados para controlar o excesso de confiança e o otimismo de gestores excessivamente qualificados.

\section{Referencial Teórico}

\section{Sofisticação financeira}

O sucesso de uma empresa pode estar relacionado com o recrutamento de profissionais talentosos, principalmente ao se tratar de indivíduos que ocupam elevados níveis hierárquicos (Dickel \& Moura, 2016). Diversos autores dão indícios da existência de um interesse das empresas na contratação de gestores com algumas características específicas, tais como: (a) formação internacional, formação financeira; (b) experiência no ramo de atividade da empresa, experiência no setor financeiro, experiência como diretor financeiro, experiência na mesma função, experiência internacional, e experiência em elevada hierarquia em outra empresa (Carr, Inkson, \& Thorn, 2005; Custódio, Ferreira, \& Matos, 2013; Dauth, Pronobis, \& Schmid, 2017; Faulconbridge, Beaverstock, Hall, \& Hewitson, 2009; Li, Sun, \& Ettredge, 2010; Li, Wei, \& Lin, 2016; Paton \& Wagner, 2014; Saxena \& Bendale, 2014; Torres \& Augusto, 2016). Oliva e Albuquerque (2007) salientam que no momento da contratação ou eleição de um indivíduo a um cargo de elevada hierarquia é levada em consideração a existência de conhecimentos relacionados a finanças. Indivíduos que almejam tal cargo, buscam desenvolver seus conhecimentos financeiros (Manfredi, 1998). Por esse ângulo, denomina-se sofisticação financeira, o acúmulo de conhecimentos advindos da formação acadêmica e profissional relacionada a finanças.

A ideia de que os indivíduos com maior sofisticação financeira tendem a tomar decisões mais acertadas, ou seja, com maior racionalidade, encontra respaldo no trabalho de Li, Wei e Lin (2016), que afirmam que os investidores institucionais e analistas de mercado consideram mais relevantes as informações divulgadas nos relatórios financeiros das empresas que possuem indivíduos com maior conhecimento e experiência nos cargos de CEO e CFO. Além disso, diversos estudos relacionam a qualidade dos relatórios financeiros com as características acadêmicas e profissionais dos gestores (Dauth et al., 2017; Li et al., 2016). 
As empresas geridas por indivíduos com maiores conhecimentos advindos da formação acadêmica e da experiência profissional, tendem a apresentar melhor desempenho (Davis, DeBode, \& Ketchen, 2013), e como contrapartida, essas empresas devem oferecer remunerações mais elevadas (Custódio et al., 2013; Trejo, Gutiérrez, \& Guzman, 2016). Por esse motivo, as empresas passaram a registrar seu capital intelectual como ativo intangível (Dickel \& Moura, 2016; Paraskevas, Altinay, McLean, \& Cooper, 2013). Assim, as empresas devem manter gestores bem qualificados e com remuneração proporcional aos seus conhecimentos, visto que esses são responsáveis pelas principais decisões de uma empresa, as quais devem maximizar o interesse dos acionistas (Trejo et al., 2016).

Aos executivos compete a tomada de decisões que ocorre, na maior parte das vezes, em caráter emergencial (Barros, 2005). Choo, Nag e Xia (2015), ao abordar da rapidez em que uma decisão é tomada, ressaltam as duas formas de pensamento descritas em Kahneman (2003, 2012), em que o sistema 1 age de modo reflexivo e intuitivo, enquanto o sistema $\mathbf{2}$ age de modo reflexivo e analítico. Assim, quando um executivo toma decisões, na maior parte das vezes, não dispõe de tempo hábil para pensar de forma analítica e, como consequência, utiliza o sistema 1 de pensamento. Destaca-se que, para o uso do sistema reflexivo e intuitivo, o executivo tende a utilizar seus conhecimentos passados, advindos da formação acadêmica, da experiência profissional, ou ainda, de ambos. Esse entendimento pode levar à reflexão de que quanto maior for a sofisticação financeira do gestor, maior é a propensão do indivíduo agir de forma intuitiva, o que pode conduzir a erros, ou seja, desvios cognitivos.

\section{Excesso de confiança e otimismo}

Os executivos enviesados não demostram dificuldades na tomada rápida de decisões, inclusive, possuem tendência de agir de forma precipitada na maior parte das vezes. Para Barros (2005) os executivos que apresentam excesso de confiança e otimismo tendem a decidir rapidamente sobre investimentos em novos projetos, se o viés for moderado, criar vantagem competitiva para a empresa, no entanto, decisões impulsivas poderão reduzir o valor da empresa, aceitando projetos ruins. Assim, as decisões variam de acordo com os traços de personalidade, que podem vir a favorecer ou prejudicar a empresa dependendo da intensidade em que o viés se manifesta.

Apesar de o excesso de confiança e o otimismo serem conceituados separadamente, no contexto de tomada de decisões a maior parte dos autores admite serem complementares, tornando improvável que ao conceituar um deles, não se esteja tratando também do outro, a menos que estejam trabalhando de forma separada as fases da tomada de decisão: julgamento, avaliação e decisão (Lambert, Bessière, \& N'Goala, 2012). Na concepção de Barros (2005), Jemaiel, Mamoghli e Seddiki (2013) o excesso de confiança pode ser entendido como a tendência de os indivíduos superestimarem suas habilidades, conhecimentos e qualidade das informações que são capazes de gerar, para embasar a tomada de decisões, além de subestimar as mesmas capacidades nos demais indivíduos. Quando o indivíduo acredita que suas probabilidades de sucesso estão acima da média e as de fracasso são quase nulas, passa a apresentar adicionalmente o viés otimismo.

Os gestores otimistas tendem a acreditar positivamente nas perspectivas futuras da empresa em que atuam, superestimando a probabilidade de sucesso dos projetos que aderirem e subestimando a probabilidade de fracasso dos mesmos (Barros, 2005). Hackbarth (2008) entende que o otimismo está relacionado à superestimação da probabilidade de futuros eventos favoráveis e o excesso de confiança está relacionado à superestimação dos conhecimentos, contudo dificilmente executivos apresentarão apenas um desses vieses, pois um provoca o outro. Por essas circunstâncias, a maior parte das métricas existentes mensura os dois vieses de forma agrupada.

Hill, Kern e White (2014) apontam sete métricas utilizadas como forma de mensuração do excesso de confiança e otimismo do executivo. Cada uma é construída por uma lógica rigorosamente fundamentada: (a) a linguagem do executivo - contagem de declarações enviesadas do executivo transmitidas na carta dos acionistas; (b) comentários da mídia - intensidade dos noticiários em relação ao viés gestor; (c) desempenho organizacional - retorno por ação após sua atuação como gestor; (d) investimentos organizacionais - o nível de investimento; (e) remuneração - os CEO’s que apresentam 
maiores capacidades são mais bem pagos, (f) exercício da opção - CEO's que não exercem suas opções de ações de forma imediata aumentam seus riscos; e (g) compras de ações - demonstra a superestimação de suas capacidades de aumentar o valor da empresa. Podem existir outras formas de mensuração para o excesso de confiança e otimismo utilizando dados secundários.

A maior parte dos estudos concentrados no excesso de confiança e otimismo dos executivos tem abordado o impacto dos vieses nas decisões a estes abrangidas, como por exemplo, endividamento (Antonczyk \& Salzmann, 2014; Barros, 2005; Hackbarth, 2009; Huang, Tan, \& Faff, 2016), investimento (Barros, 2005; Lambert et al., 2012; Wang, Chen, Chen, \& Huang, 2016), dividendos (Deshmukh, Goel, \& Howe, 2013) e recompra de ações (Andriosopoulos, Andriosopoulos \& Hoque, 2013). Esses estudos comprovam que a mensuração dos vieses cognitivos excesso de confiança e otimismo dos executivos na maior parte das vezes se dá de forma indireta, utilizando dados secundários de acordo com a lógica expressa na literatura, conforme já destacado por Fellner e Krügel (2012). Por vezes, a maneira com que o CEO procede em relação à suas escolhas, pode indicar a presença dos vieses cognitivos excesso de confiança e otimismo conjuntamente, devendo as métricas utilizadas considerar esta condição.

A presente pesquisa trabalha de forma conjunta os conceitos excesso de confiança e otimismo, pois de acordo com Gervais, Hearton e Odean (2003), é raro encontrar um Diretor Presidente que obtenha apenas um desses traços de personalidade. Hill et al. (2014) revelam que alguns estudiosos também se referem a esses vieses como viés da arrogância, devido aos efeitos negativos das características psicológicas dos gestores de elevada hierarquia. Por esse ângulo Bollaert e Petit (2010) perceberam que os estudos têm dedicado esforços principalmente em identificar efeitos negativos da arrogância, no entanto, alguns aspectos desses desvios cognitivos são socialmente desejáveis, sendo os efeitos positivos uma fértil linha de pesquisa. Além disso, destacam que se tem estudado o impacto da arrogância em diferentes contextos de forma negativa ou positiva por algumas poucas vezes, mas há negligência dos pesquisadores em não considerar as características que a causam ou agravam. Em relação à necessidade de haver estudos que procurassem identificar as causas do excesso de confiança, Mishra e Metilda (2015) estudaram o impacto da experiência de investimento e o nível de instrução dos investidores sobre esse viés, mas não consideraram o otimismo. Nesse contexto, a presente pesquisa busca contribuir com essa lacuna existente, considerando que os CEO's podem apresentar ambos os desvios cognitivos.

Bollaert e Petit (2010) destacam que diversos estudos têm buscado analisar o impacto do excesso de confiança e otimismo em diferentes aspectos que impactam na economia, mas que há a necessidade de estudos que busquem encontrar características que causam ou agravam esses vieses. Nesse sentido, Mishra e Metilda (2015) desenvolveram na tentativa de inferir que a experiência de investimento, o nível de instrução dos investidores e o gênero podem estar associados ao excesso de confiança. Outros autores também dão indícios de que características que são consideradas no presente trabalho entre os componentes da Sofisticação Financeira, são desejadas nos CEO's e podem contribuir para os desvios cognitivos excesso de confiança e otimismo, tendo como exemplo: o nível de escolaridade; conhecimentos acadêmicos nas áreas de administração, contabilidade, economia e finanças; formação e/ou experiência internacional anos de experiência no mesmo setor; anos de experiência no mesmo cargo; experiência de atuação anterior como diretor financeiro; exercer outros cargos de elevada hierarquia em outras empresas ao mesmo tempo em que se exerce o cargo de CEO (Barros, 2005; Carr et al., 2005; Custódio et al., 2013; Davis et al., 2013; Dauth et al., 2017; Faulconbridge et al., 2009; Li et al., 2010; Li et al., 2016, Oliva \& Albuquerque, 2007; Torres \& Augusto, 2016). Nesse sentido, pretende-se com este trabalho responder à seguinte hipótese teste:

$\mathbf{H}_{1}$ : A Sofisticação Financeira do CEO está relacionada positiva e significativamente ao excesso de confiança e otimismo. 


\section{Metodologia}

A população da pesquisa compreende empresas de capital aberto listadas na BM\&FBovespa durante o período de 2011 a 2015, o que corresponde a 418 empresas. Ao efetuar a coleta de dados observou-se que em diversas empresas, seus diretores não efetuaram ao menos uma transação de compra ou venda de ações (dados necessários para mensuração da variável excesso de confiança e otimismo) durante todo o período de abrangência desta pesquisa. Acredita-se que pode haver restrições internas ou externas nas empresas que desincentivem a negociação (uma vez que tais transações poderiam influenciar na decisão dos interessados), assim, dado que a impossibilidade de transacionar ações poderia enviesar as análises, entendeu-se adequado desconsiderar da amostra as empresas que não tenham realizado pelo menos uma transação de compra ou venda de ações entre 2011 e 2015, de forma a considerar apenas empresas em que esta prática ocorre ao menos uma vez entre o período analisado. Assim, é assegurado que a empresa não restringe transações de compra e/ou venda de ações pelos diretores, e consequentemente, não havendo transação para os demais períodos abrangidos na pesquisa, considera-se que o CEO obtinha a possibilidade de negociar ações da empresa e não a fez.

A composição da amostra, de acordo com as exclusões necessárias pelas variáveis do modelo econométrico, está descrita na Tabela 1, apresentada a seguir:

Tabela 1

\section{Composição da Amostra}

\begin{tabular}{|c|c|c|c|c|c|c|c|c|c|c|}
\hline \multirow[t]{2}{*}{ População } & \multicolumn{5}{|c|}{ Empresas } & \multicolumn{5}{|c|}{ Percentual } \\
\hline & 2011 & 2012 & 2013 & 2014 & 2015 & 2011 & 2012 & 2013 & 2014 & 2015 \\
\hline $\begin{array}{l}\text { Empresas listadas na } \\
\text { BM\&FBovespa em } \\
\text { 31/12/2015 }\end{array}$ & 418 & 418 & 418 & 418 & 418 & $100 \%$ & $100 \%$ & $100 \%$ & $100 \%$ & $100 \%$ \\
\hline Seleção da amostra & \multicolumn{5}{|c|}{ Empresas excluídas } & \multicolumn{5}{|c|}{ Percentual } \\
\hline $\begin{array}{l}\text { Exclusão por não } \\
\text { apresentarem compra ou } \\
\text { venda de ações ao menos em } 1 \\
\text { período }\end{array}$ & 229 & 229 & 229 & 229 & 229 & $54,78 \%$ & $54,78 \%$ & $54,78 \%$ & $54,78 \%$ & $54,78 \%$ \\
\hline
\end{tabular}

\begin{tabular}{lcccccccccc}
\hline Amostra inicial & $\mathbf{1 8 9}$ & $\mathbf{1 8 9}$ & $\mathbf{1 8 9}$ & $\mathbf{1 8 9}$ & $\mathbf{1 8 9}$ & $\mathbf{4 5 , 2 2 \%}$ & $\mathbf{4 5 , 2 2 \%}$ & $\mathbf{4 5 , 2 2 \%}$ & $\mathbf{4 5 , 2 2 \%}$ & $\mathbf{4 5 , 2 2 \%}$ \\
\hline $\begin{array}{l}\text { Exclusão por não haver } \\
\text { disponível o último preço da } \\
\text { ação do período }\end{array}$ & 19 & 15 & 9 & 11 & 13 & $4,55 \%$ & $3,59 \%$ & $2,15 \%$ & $2,63 \%$ & $3,11 \%$ \\
\hline $\begin{array}{l}\text { Exclusão por ter fechado o } \\
\text { capital durante o período de } \\
\text { coleta }\end{array}$ & 2 & 2 & 2 & 2 & 2 & $0,48 \%$ & $0,48 \%$ & $0,48 \%$ & $0,48 \%$ & $0,48 \%$ \\
\hline $\begin{array}{l}\text { Exclusão por não ter } \\
\text { divulgado o formulário de } \\
\text { referência }\end{array}$ & - & - & - & 1 & 1 & $0,00 \%$ & $0,00 \%$ & $0,00 \%$ & $0,24 \%$ & $0,24 \%$ \\
\hline $\begin{array}{l}\text { Exclusão das empresas que } \\
\text { não divulgaram o ROA por 5 } \\
\text { exercícios anteriores }\end{array}$ & 21 & 14 & 13 & 6 & 2 & $5,02 \%$ & $3,35 \%$ & $3,11 \%$ & $1,44 \%$ & $0,48 \%$ \\
\hline $\begin{array}{l}\text { Total de exclusões } \\
\text { Amostra final }\end{array}$ & $\mathbf{2 7 1}$ & $\mathbf{2 6 0}$ & $\mathbf{2 5 3}$ & $\mathbf{2 4 9}$ & $\mathbf{2 4 7}$ & $\mathbf{6 4 , 8 3 \%}$ & $\mathbf{6 2 , 2 0 \%}$ & $\mathbf{6 0 , 5 3 \%}$ & $\mathbf{5 9 , 5 7 \%}$ & $\mathbf{5 9 , 0 9 \%}$ \\
\hline
\end{tabular}

Nota. Fonte: Elaborado pelos autores. 
Conforme observado na Tabela 1, as amostras são distintas para cada um dos períodos abrangidos por este estudo. Contudo, optou-se por proceder dessa forma em relação às exclusões, a fim de manter maior quantidade de observações possíveis, e garantir a relevância desse estudo ao mercado brasileiro. Assim, as empresas que compreendem cada um dos períodos da amostra são dispares, sendo que a amostra inicial era composta de 189 empresas distintas e 326 CEO's, e após as exclusões mantiveramse 179 empresas distintas e 298 CEO's.

A fim de tratar estatisticamente os dados para encontrar resposta à hipótese de que a sofisticação financeira do CEO está relacionada ao excesso de confiança e otimismo, bem como verificar as demais relações teóricas entre os vieses cognitivos e as variáveis de controle, utilizou-se de uma regressão linear múltipla, descrita nas equações (a) que possui como variável independente o Índice de Sofisticação Financeira, e (b) que possui como variáveis independentes a Sofisticação Financeira segregada em Índice de Sofisticação Financeira Acadêmica e Índice de Sofisticação Financeira Profissional:

$$
\begin{aligned}
& E C O_{i, t}=\beta_{0}+\beta_{1} I_{S F_{i, t}}+\beta_{2} \text { CEOE }_{i, t}+\beta_{3} I_{i, t}+\beta_{4} G_{i, t}+\beta_{5} \text { Troc }_{i, t}+\beta_{6} \text { CEOPCA }_{i, t}+ \\
& \beta_{7} T_{i, t}+\beta_{8} I P L_{i, t}+\beta_{9} R_{i, t}+\beta_{10} P I B_{i, t-1}+\varepsilon \\
& E C O_{i, t}=\beta_{0}+\beta_{1} I S F A_{i, t}+\beta_{2} I S F P_{i, t}+\beta_{3} \text { CEOE }_{i, t}+\beta_{4} I_{i, t}+\beta_{5} G_{i, t}+\beta_{6} \text { Troc }_{i, t}+ \\
& \beta_{7} C E O P C A_{i, t}+\beta_{8} T_{i, t}+\beta_{9} Q P A_{i, t}+\beta_{10} R_{i, t}+\beta_{11} P I B_{i, t-1}+\varepsilon
\end{aligned}
$$

Em que:

$E C O_{i, t}-$ Excesso de Confiança e Otimismo da empresa $i$ no ano $t$

$\beta_{0}, \beta_{1}, \beta_{2}, \beta_{3}, \beta_{4}, \beta_{5}, \beta_{6} \beta_{7}, \beta_{8}, \beta_{9}, \beta_{10}, \beta_{11}$ - Coeficientes do modelo de regressão;

$I S F_{i, t}$ - Índice de Sofisticação Financeira da empresa $i$ no ano $t$;

$I S F A_{i, t}$ - Índice de Sofisticação Financeira Acadêmica da empresa $i$ no ano $t$;

$I S F P_{i, t}$ - Índice de Sofisticação Financeira Profissional da empresa $i$ no ano $t$;

$C E O E_{i, t}-$ CEO Empreendedor da empresa $i$ no ano $t$;

$I_{i, t}$ - Idade do CEO da empresa $i$ no ano $t$;

$G_{i, t}-$ Gênero do CEO da empresa $i$ no ano $t$;

$\operatorname{Troc}_{i, t}-$ Troca de CEO da empresa $i$ no ano $t$

CEOPCA $_{i, t}-$ CEO e Presidente do Conselho da Administração da empresa $i$ no ano $t$;

$T_{i, t}$ - Tamanho da empresa $i$ no ano $t$;

$I P L_{i, t}-$ Índice $\mathrm{P} / \mathrm{L}$ da empresa $i$ no ano $t$;

$R_{i, t}-$ Risco da empresa $i$ no ano $t$;

$P I B_{i, t-1}$ - Produto Interno Bruto da empresa $i$ no ano $t$;

$\varepsilon_{i, t}-$ Termo de erro da regressão.

Variável dependente - Excesso de Confiança e Otimismo: O CEO excessivamente confiante e otimista, acredita que as ações disponíveis para negociação estão subvalorizadas, e que a sua gestão pode contribuir para atingir valores superiores, pois confia em suas habilidades, conhecimentos e capacidades de gerar informações úteis, melhorando o desempenho da empresa. Além disso, ele acredita ainda que é mais propenso a resultados positivos, subestimando os riscos (de não diversificar). Esse 
raciocínio é válido tanto para compra de ações, como sobre o recebimento de remuneração por opções de ações, uma vez que fica a critério do CEO a execução da compra. No Brasil, as poucas empresas utilizam desse incentivo, concedem opções de ações europeias com algumas restrições quando o prazo mínimo em que a ação deve ficar na posse do gestor (vesting). Também, é possível observar que as empresas brasileiras que tem concedido esse incentivo, tem mantido essa prática ao longo do tempo. Como ocorre, por exemplo, na remuneração de executivos da Grendene SA, o que pode ser observado no Formulário de referência: item 13. Remuneração de administradores e subitem 13.5 Remuneração baseada em ações ([B] $]^{3}$ - Brasil, Bolsa e Balcão, 2017). Assim, se o executivo apresentar excesso de confiança e otimismo, haverá mais exercício de opções do que venda, e caso não haja excesso de confiança e otimismo haverá mais vendas das ações que saíram do vesting. Malmendier e Tate (2005, 2008) destacam que quando os CEO's adquirem maiores quantidades de ações do que vendem dentro de um período de tempo, transparecem esses vieses.

No contexto brasileiro, os dados de compra e venda de ações dos CEO's não são divulgados individualmente, sendo disponibilizada a informação conjuntamente com os demais diretores. Dessa forma, cabe utilizar esses dados como proxy para mensurar os vieses excesso de confiança e otimismo do CEO (um argumento fundamental aqui é o reconhecimento da influência do CEO sobre os demais diretores). Considerando que existe uma elevada dispersão entre o saldo de compra e venda de ações para cada uma das empresas, considerou-se mais adequado trabalhar com a ponderação da compra sobre a venda ocorridas dentro de um mesmo período de tempo. Conforme pode ser visualizado na equação (3), a seguir:

$$
\mathrm{ECO}_{\mathrm{i}, \mathrm{t}}=\frac{\mathrm{C}_{\mathrm{i}, \mathrm{t}}}{\mathrm{V}_{\mathrm{i}, \mathrm{t}}}
$$

Em que:

$$
\begin{aligned}
& E C O_{i, t}-\text { Excesso de Confiança e Otimismo do CEO da empresa } i \text { no ano } t \\
& C_{i, t} \text { - Compra de ações pelo CEO referente à empresa em que atua }(i) \text { no ano } t \\
& V_{i, t} \text { - Venda de ações pelo CEO referente à empresa em que atua }(i) \text { no ano } t
\end{aligned}
$$

Variável Independente - Sofisticação Financeira:A Sofisticação Financeira pode levar um gestor a apresentar os vieses cognitivos excesso de confiança e otimismo. Complementarmente à hipótese levantada nesse estudo, Kahneman (2012) argumenta que a crença excessiva dos investidores em relação aos conhecimentos e habilidades do CEO faz com que este superestime ainda mais suas capacidades e subestimem os riscos, tornando-o excessivamente confiante e otimista. Entende-se que um gestor pode ser sofisticado financeiramente, quando há o acúmulo de conhecimentos financeiros advindos da formação acadêmica e experiência profissional. Nesse sentido, a mensuração da variável se dá pela formação de um Índice de Sofisticação Financeira (ISF), que contempla de forma conjunta aspectos acadêmicos e profissionais já destacados por outros autores. Além disso, compreende-se necessário avaliar separadamente a dimensão acadêmica da dimensão profissional, formando o Índice de Sofisticação Acadêmica (ISFA) e o Índice de Sofisticação Profissional (ISFP), uma vez que ao analisar as dimensões de forma conjunta, pode ocorrer a anulação da significância em função da importância individual da dimensão em relação ao índice. Os índices variam entre $\mathbf{0}$ e $\mathbf{1}$, dependendo da ocorrência do item e da pontuação atribuída ao mesmo, dado ao grau de importância distintos detectados na Análise de Componentes Principais (ACP).

A Sofisticação Financeira propõe a existência das dimensões acadêmica e profissional. Para a mensuração da dimensão acadêmica foram considerados os itens: Formação Internacional (FI) (se possui formação acadêmica no nível de graduação e/ou pós-graduação em universidades internacionais) e Formação Financeira (FF) (se possui formação acadêmica no nível de graduação e/ou pós-graduação relacionada à finanças, ou seja, cursos de Administração, Contabilidade, Economia ou Finanças). Já, para a mensuração da dimensão profissional foram considerados os itens: Experiência no Ramo (ER) (quantidade de anos com experiência profissional no mesmo ramo da empresa em que atua), Experiência no Setor Financeiro (ESF) (se possui experiência profissional em empresas do setor financeiro), 
Experiência como Diretor Financeiro (EDF) (se possui experiência profissional como Diretor Financeiro), Experiência como CEO - ECEO (quantidade de anos que já exerceu o cargo de CEO na empresa em que atua), Experiência Internacional (EI) (se possui experiência profissional em empresas internacionais) e Elevada Hierarquia em Outra Empresa (EHOE) (se exerce função de elevada hierarquia em outra empresa ao mesmo tempo em que exerce a função de CEO). Tais informações foram coletadas no resumo do currículo do CEO disponível no Formulário de Referência (itens 12.6 e 12.8), resumo do currículo do CEO disponível no IAN - Informações Anuais, site da empresa, Bloomberg, Linkedin, currículo Lattes, noticiários e revistas que possam ter realizado entrevistas ou ter publicado notícias relacionadas ao CEO (G1, O Globo, Veja, Valor, Exame e Estadão).

A Análise de Componentes Principais (ACP) confirmou a existência de 2 grupos (ou seja, duas dimensões), contudo, com duas variações: (a) a realocação do item ER para a dimensão acadêmica, e a eliminação do item EHOE do índice. A partir da ACP, foi possível atribuir pesos distintos para as dimensões e seus respectivos itens. A dimensão acadêmica representa 50,52\% do índice (sendo que: FI - 18,62\%; FF - 18,07\%; e ER - 13,83\%), e a dimensão profissional representa os demais $49,48 \%$ do índice (sendo que: ESF - 10,87\%; EDF - 15,21\%; ECEO - 12,27\%; e EI - 11,13\%). Cabe destacar que, para a formação do índice, os itens mensurados de forma dicotômica tiveram a atribuição do percentual destacado, quando a ocorrência do fato, e $0 \%$ no caso de não ocorrência. Já, os itens mensurados em anos, como é o caso dos itens: ER (0 anos - 0\%; 1 a 10 anos - 2,77\%; 11 a 20 anos $-5,53 \% ; 21$ a 30 anos - 8,30\%; 31 a 40 anos - 11,06; e 41 ou mais anos - 13,83\%) e ECEO ( 0 anos - 0\%; 1 a 10 anos $4,09 \%$; 11 a 20 anos $-8,18 \%$; e 21 ou mais anos - 12,27\%), obtiveram tratamento diferenciado. Para a formação do ISFA e ISFP foi necessário efetuar a readequação dos percentuais dos itens de forma proporcional, considerando que a dimensão equivale a 100\% do índice.

Variável de controle - CEO Empreendedor (CEOE): No momento em que o empreendedor (fundador ou herdeiro), atua como CEO de sua própria empresa, aumenta o risco idiossincrático, já que suas fontes de rendimento possuem a mesma origem (Barros, 2005; Bharati, Doellman, \& Fu, 2016). No entanto, acreditam ter diminuído seus riscos, já que julgam possuir o controle da situação, superestimando seus conhecimentos e habilidades (Kahneman, 2012). Dessa forma, foi controlado esse efeito por intermédio de uma variável binária, atribuindo 1 para as empresas obtiverem CEO's fundadores ou herdeiros, e 0 para as empresas que não apresentarem essas características de gestão. Os dados foram coletados manualmente, efetuando uma busca nos sites já referenciados no tópico anterior, pelo nome CEO.

Variável de controle - Presidente do Conselho da Administração (CEOPCA): O CEO é responsável pelas decisões imediatas de uma empresa, contudo é provável que em algumas empresas, principalmente em que o Presidente do Conselho da Administração (PCA) é o fundador ou controlador do negócio, as decisões de maior competência sejam atribuídas a este cargo ao invés do CEO. Assim, quando o CEO assume ao mesmo tempo o cargo de PCA, tende a ser excessivamente confiante e otimista, uma vez que não há outra função de elevada hierarquia capaz de anular suas decisões viesadas (Barros, 2005). Esse efeito será controlado por uma variável dicotômica, atribuindo 1 quando o CEO também exerce o cargo de PCA, e 0 quando não exercer.

Variável de controle - Troca de CEO (Troc): A troca do CEO é um evento amplamente estudado pela literatura financeira, devido a resultar em diversos impactos positivos e negativos na economia (Dedman \& Lin, 2002; Lin \& Liu, 2012). Acredita-se que a troca do CEO proporciona resultados positivos, pois impossibilita a inercia da empresa, mantendo-a em constante adaptação, logo, podendo eliminar a existência de um viés (Barros, 2005). A variável foi mensurável por uma dummy, atribuindo 1 quando houve troca de CEO dentro do período.

Variável de controle - Gênero (G): Diversos estudos têm mostrado que o grau de excesso de confiança e otimismo apresentam-se em diferentes proporções dependendo do gênero (Mishra \& Metilda, 2015). Os autores explicam que o indivíduo de gênero masculino tem mais propensão a apresentar esses vieses por possuírem maiores experiências profissionais e por receberem maior feedback. Huang e Kisgen (2013) revelam que o número de CEO's do gênero feminino tem aumentado, que são menos propensos a fazer aquisições e emitem menos dívidas. Considerando que os resultados 
de diversas pesquisas convergem com a ideia de que os CEO's do gênero masculino possuem maior tendência a apresentar excesso de confiança e otimismo, identifica-se a necessidade de controlar essa variável dicotômica, sendo representado por 1 as empresas geridas por CEO gênero feminino e 0 para as empresas geridas por CEO de gênero masculino.

Variável de controle - Idade (I): Mishra e Metilda (2015) mencionam que a experiência profissional pode levar o CEO a apresentar excesso de confiança e otimismo, já o trabalho de Yim (2013) propõe que CEO's mais novos possuem propensão a apresentar tais vieses, contudo, para que se adquira experiência é necessária dedicação de algum período de tempo, de forma que o indivíduo tenderia a ter idade mais elevada. Essa variável é mensurada pela idade em anos.

Variável de controle - Tamanho da empresa (T): Hirshleifer, Low e Teoh (2012) e Bharati, Doellman e Fu (2016), compreendem que alguns desvios cognitivos, como é o caso do excesso de confiança e otimismo, podem ser característicos de executivos que beneficiem os acionistas, dado que possuem uma maior tendência a investir em projetos arriscados, chamando assim a atenção de investidores. Essa variável é mensurada pelo logaritmo natural do ativo da empresa.

Variável de controle - Índice P/L (IPL): Considerando a proxy utilizada para mensurar o ECO, o índice $\mathrm{P} / \mathrm{L}$ poderia motivo plausível para o CEO adquirir ações da própria empresa. Ao comparar o resultado da ponderação de diversas empresas, é possível obter argumentos consistentes para escolher um investimento (Aguiar, Sales, \& Sousa, 2008; Kimura, 2003), pois este demonstra em quantos anos haveria recuperação o dinheiro investido.

Variável de controle - Risco (R): No momento em que um indivíduo decide efetuar investimentos no mercado de ações, é natural que o mesmo tenha a preocupação de dimensionar o risco do negócio, podendo para isso, valer-se de dados contábeis disponíveis. Utilizou-se como medida de risco o desvio padrão do ROA dos 5 anos anteriores em consonância com Dalmácio, Lopes, Rezende e Sarlo (2013), e Gatsios (2013). Quanto maior a dispersão dos retornos, mais elevados são os riscos que um indivíduo corre ao adquirir ações da empresa que possui tal característica. Ao considerar que o CEO excessivamente confiante e otimista não possui aversão ao risco, a tendência é que o mesmo adquira as ações em momentos de maior volatilidade.

Variável de controle - Produto Interno Bruto do ano anterior (PIB t-1): Em períodos em que a macroeconomia se demonstra promissora, os indivíduos que obtiverem reservas de capital tendem a utilizar uma parcela destas para efetuar investimentos. Um bom momento macroeconômico pode ser percebido de acordo com o crescimento do Produto Interno Bruto (PIB) (informação anual fornecida pelo IBGE) do ano anterior, pois este representa em valores o bem estar da população em geral, uma vez que esse percentual considera a produção de bens e serviços e a demanda para um país em um dado período de tempo (Lélis, Bredow, \& Cunha, 2015). Assim, um CEO excessivamente confiante e otimista tende a investir após um período de baixo crescimento do PIB, por acreditar que um momento de baixa na macroeconomia atrelado ao seu potencial como gestor, pode vir a aumentar o valor da empresa futuramente.

\section{Análise dos Resultados}

Com intuito de verificar se as variáveis independentes contribuem com a explicação da variável dependente, optou-se por utilizar uma regressão múltipla buscando obter maior eficiência em captar as relações esperadas, uma vez que é possível reduzir o erro inserindo variáveis de controle (Field, 2009). Para tanto, torna-se necessário efetuar alguns testes de pressupostos de uma regressão múltipla, para verificar se os dados permitem a utilização desse tipo de análise, conforme exposto na Tabela 2: 
Tabela 2

\section{Pressupostos da Regressão}

\begin{tabular}{llllllllll}
\hline \multirow{2}{*}{ Modelos } & \multicolumn{2}{l}{ Durbin-watson } & White & \multicolumn{3}{c}{ Normalidade dos Resíduos } & VIF & Teste F \\
\cline { 2 - 9 } & Coef. & p-valor & Coef. & p-valor & Coef. & p-valor & $\leq$ & p-valor \\
\hline ISF & 1,66 & 0,81 & 37,64 & 0,99 & 66447,00 & $0,00 * * *$ & 1,208 & $0,00^{* * * *}$ \\
\hline ISFA e ISFP & 1,68 & 0,88 & 87,65 & 0,10 & 63222,10 & $0,00 * * *$ & 1,208 & $0,00^{* * * *}$ \\
\hline
\end{tabular}

Nota. Excesso de Confiança e Otimismo (ECO) -: Índice Compra/Venda; Índice de Sofisticação Financeira (ISF): formado por dimensão acadêmica e dimensão profissional; Índice de Sofisticação Financeira Acadêmica (ISFA): apenas dimensão acadêmica; Índice de Sofisticação Financeira Profissional (ISFP): Apenas dimensão profissional. Fonte: Elaborado pelos autores.

*** Significante ao nível de 1\%; ** Significante ao nível de 5\%; * Significante ao nível de 10\%.

Conforme apresentado na Tabela 2, foram testados os pressupostos de uma análise multivariada para dois modelos econométricos: (a) ISF; (b) e ISFA e ISFP. O teste de Durbin-Watson apontou ausência de autocorrelação tanto para o modelo ISF (Coef. - 1,66; p-valor 0,81), quanto para o modelo ISFA e ISFP (Coef. - 1,68; p-valor 0,88). O teste de White indicou a ausência de heterocedasticidade (ISF: p-valor 0,99; ISFA e ISFP: p-valor 0,10), e O teste de Fatores de Inflacionamento da Variância, apresentou ausência de problemas de multicolinearierade (VIF $\leq 1,208$ ). Por sua vez, apesar de o teste de normalidade dos resíduos ter rejeitado a hipótese nula de normalidade, conforme Wooldridge (2005) um grande número de observações (810 neste trabalho) faz com que seja satisfeita a normalidade assintótica dos estimadores OLS de acordo com o Teorema Central do Limite. Assim, considera-se que os pressupostos são adequados para conduzir a análise da regressão a fim de encontrar argumentos mais sólidos e responder à hipótese levantada, conforme Tabela 3, a seguir:

Tabela 3

\section{Regressões Lineares Multivariadas}

\begin{tabular}{lllllll} 
& \multicolumn{2}{l}{ Modelo ISF } & \multicolumn{3}{c}{ Modelo ISFA e ISFP } \\
\cline { 2 - 8 } Variável & Coef. & $\begin{array}{l}\text { Erro } \\
\text { Padrão }\end{array}$ & p-valor & Coef. & $\begin{array}{l}\text { Erro } \\
\text { Padrão }\end{array}$ & p-valor \\
\hline Constante & $-14,01$ & 8,11 & $0,08^{*}$ & $-16,47$ & 2,59 & $0,04^{* *}$ \\
\hline ISF & 0,07 & 0,07 & 0,26 & - & & - \\
\hline ISFA & - & & - & 0,12 & 0,05 & $0,01^{* *}$ \\
\hline ISFP & - & & - & $-0,07$ & 0,06 & 0,19 \\
\hline CEOE & $-1,46$ & 2,60 & 0,58 & $-1,37$ & 2,59 & 0,60 \\
\hline I & 0,08 & 0,11 & 0,47 & 0,12 & 0,11 & 0,28 \\
\hline G & 23,31 & 6,80 & $0,00^{* * *}$ & 21,28 & 6,84 & $0,00^{* * *}$ \\
\hline Troc & $-4,11$ & 2,82 & 0,14 & $-4,20$ & 2,81 & 0,14 \\
\hline CEOPCA & $-1,88$ & 3,43 & 0,58 & $-1,77$ & 3,42 & 0,60 \\
\hline T & 1,57 & 0,56 & $0,00^{* * *}$ & 1,49 & 0,56 & $0,01^{* *}$ \\
\hline QPA & $-0,01$ & 0,01 & 0,54 & $-0,01$ & 0,01 & 0,52 \\
\hline R & $-0,00$ & 0,00 & 0,92 & $-0,00$ & 0,00 & 0,80 \\
\hline PIB & $-0,54$ & 0,43 & 0,21 & $-0,48$ & 0,43 & 0,26 \\
\hline
\end{tabular}


Tabela 3 (continuação)

\begin{tabular}{|c|c|c|c|c|c|c|}
\hline \multirow[b]{3}{*}{ Variável } & \multirow{2}{*}{\multicolumn{3}{|c|}{ Modelo ISF }} & \multirow{2}{*}{\multicolumn{3}{|c|}{ Modelo ISFA e ISFP }} \\
\hline & & & & & & \\
\hline & Coef. & $\begin{array}{l}\text { Erro } \\
\text { Padrão }\end{array}$ & p-valor & Coef. & $\begin{array}{l}\text { Erro } \\
\text { Padrão }\end{array}$ & p-valor \\
\hline E. P. variável dependente & 30,22 & & & 30,22 & & \\
\hline E. P. da regressão & 29,87 & & & 29,78 & & \\
\hline R-quadrado & $3,53 \%$ & & & $4,22 \%$ & & \\
\hline R-quadrado ajustado & $2,32 \%$ & & & $2,90 \%$ & & \\
\hline Teste F & 2,92 & $0,00 * * *$ & & 3,20 & $0,00 * * *$ & \\
\hline
\end{tabular}

Nota. Excesso de Confiança e Otimismo (ECO): Índice Compra/Venda; Índice de Sofisticação Financeira (ISF); Índice de Sofisticação Financeira Acadêmica (ISFA): apenas dimensão acadêmica; ISFP - Índice de Sofisticação Financeira Profissional: Apenas dimensão profissional; Idade (I): idade em anos; Tamanho da empresa (T): calculado pelo logaritmo natural do ativo; Índice P/L (IPL): calculado pela ponderação Preço/Lucro; Risco (R): calculado pelo desvio padrão do ROA dos 5 anos anteriores; Produto Interno Bruto (PIB): percentual do crescimento do PIB informado pelo IBGE; CEOE - CEO Empreendedor: É CEO e fundador ou herdeiro do negócio 1, caso contrário 0; Gênero (G): Mulher 1 e homem 0; Troca de CEO (Troc): Existe troca de CEO durante o período 1, caso contrário 0; CEOPCA - CEO e Presidente do Conselho da Administração: Atua como CEO e como PCA da mesma empresa concomitantemente 1, caso contrário 0. Fonte: Elaborado pelos autores. *** Significante ao nível de 1\%; ** Significante ao nível de 5\%;* Significante ao nível de $10 \%$.

A regressão (Tabela 3), revelou que o ISF não possui poder explicativo em relação a variável dependente ECO, uma vez que não houve significância (coef. 0,07; p-valor 0,26). Esse fato contraria alguns indícios detectados na literatura, como Kahneman (2012), que ressalta que a crença excessiva dos acionistas na Sofisticação Financeira do CEO, poderia levar esse profissional a superestimar ainda mais suas capacidades e subestimarem seus riscos. Por outro lado, há trabalhos que sinalizam que a formação acadêmica e a experiência profissional são desejados pelos acionistas que visam à maximização de sua utilidade (Dickel \& Moura, 2016; Grip \& Sauermann, 2013).

Por sua vez, quando consideradas as dimensões separadamente, formando dois índices (modelo 2), foi possível observar que o ISFA apresentou significância a um nível de 5\%, tangenciando o nível de $1 \%$ (coef. 0,12; p-valor 0,01), indicando que uma maior formação acadêmica explica a presença dos vieses cognitivos ECO; por outro lado, o ISFP não apresentou significância estatística (coef. -0,07; pvalor 0,19$)$.

Com o intuito de proporcionar uma interpretação econômica dos testes, foi rodado o mesmo modelo econométrico com equação Log-Log, ou seja, logaritmo natural do ECO, ISFP e ISFA. O objetivo foi o de computar a elasticidade de ECO em relação aos ISFP e ISFA. O modelo apresentou para a variável ISFA um coeficiente significativo de 0,15 , sugerindo que a uma alteração de $1 \%$ no índice de sofisticação acadêmica do CEO aumentaria em 15\% o comportamento de confiança excessiva do mesmo, tudo o mais permanecendo constante.

Mishra e Metilda (2015) realizaram um estudo com intuito de identificar o impacto da experiência com investimentos, gênero e nível de escolaridade nos vieses excesso de confiança e otimismo, sendo esta a única pesquisa encontrada, que utiliza esses vieses como variável dependente, concluindo que o tempo de experiência dos investidores explica a presença dos vieses excesso de confiança e otimismo, uma vez que foi possível encontrar diferença significativa entre as respostas dos grupos de investidores e investidores experientes; além disso, constataram que o nível de excesso de confiança e otimismo aumenta de acordo com o nível de escolaridade.

O presente estudo, entretanto, apesar de assemelhar-se ao estudo de Mishra e Metilda (2015), uma vez que também apresentam o raciocínio de que a experiência e a escolaridade podem levar um indivíduo a apresentar o excesso de confiança e otimismo, diferencia-se daquele trabalho em aspectos como a amostra, e a formação de um índice (ISF), o qual considera diversos itens em relação às dimensões acadêmica e profissional relacionados a finanças. 
Considerando a composição do ISFA, pode-se dizer que há uma parcela de experiência profissional que contribui para que o CEO apresente os vieses ECO, já que na análise de componentes principais a experiência no ramo de atuação (ER) aderiu ao índice de sofisticação acadêmica. Tal fato corrobora os resultados de Mishra e Metilda (2015). Já o ISFP não apresentou significância, ou seja, o fato do CEO possuir experiência no setor financeiro (ESF), experiência como diretor financeiro (EDF), experiência como CEO da empresa atual (ECEO), e experiência internacional (EI) não contribuiu para que ele apresentasse os desvios cognitivos estudados.

Mishra e Metilda (2015) verificaram ainda que os homens apresentaram maior excesso de confiança e otimismo do que as mulheres. Contudo, o presente trabalho apontou que as mulheres têm maior ECO em relação aos homens (Modelo ISF: coef. 23,31 e p-valor 0,000; Modelo ISFA e ISFP: coef. 21,28 e p-valor 0,000). Apesar da significância obtida em ambos os modelos testados, demonstrando maior excesso de confiança e otimismo por parte das mulheres, deve-se considerar que na amostra do estudo cerca de 97,5\% dos CEO's das empresas são do gênero masculino, o que relativiza os achados relativos a gênero.

Alguns estudiosos levantam indícios de que as empresas de maior tamanho tendem a efetuar contratações de CEO's com ECO, uma vez que esses poderiam atender aos interesses dos acionistas (Hirshleifer, Low, \& Teoh, 2012). As relações entre essas variáveis, apresentaram-se significantes e positivamente relacionadas (ISF: coef. 1,57 e p-valor 0,000; ISFA e ISFP: coef. 1,49 e p-valor 0,000), conforme era esperado, indicando que quanto maior é o tamanho da empresa, maior é o excesso de confiança e otimismo do CEO. De outra forma, pode-se supor ainda que, quanto maior for o tamanho da empresa, menores podem ser as restrições internas em relação à compra e venda de ações da empresa pelo CEO, permitindo que o excesso de confiança e otimismo seja revelado no comportamento da compra de ações da empresa.

O CEO empreendedor (CEOE) é caracterizado com ECO, uma vez que parte de seu capital está investido na empresa em que atua, contrariando a lógica de diversificação de carteiras (Barros, 2005; Bharati et al. 2016). Esse entendimento é tão consolidado que, os CEO's caracterizados como empreendedores são utilizados como proxy para os vieses cognitivos ECO. Apesar disso, ao considerar essa uma variável de controle, não foi encontrada significância estatística (ISF: coef. - 1,46 e p-valor 0,58; ISFA e ISFP: coef. - 1,37 e p-valor 0,60).

Pesquisas anteriores têm mostrado resultados díspares quanto à inclinação da variável idade do CEO ser positiva ou negativa em relação ao ECO (Mishra \& Metilda, 2015; Yim, 2013). Apesar de a relação esperada ser a de que quanto maior a idade do CEO, maiores seriam suas experiências, gerando uma relação positiva com o ECO, os resultados não apontaram significância estatística (ISF: coef. 0,08 e p-valor 0,47; ISFA e ISFP: coef. 0,12 e p-valor 0,28).

O estudo também controlou a troca do CEO, em razão de haver a interpretação de que poder-seia ocorrer quebras nos comportamentos dos profissionais que exerciam a função anteriormente. Não foi identificada significância estatística para esta relação (ISF: coef. -4,11 e p-valor 0,14; ISFA e ISFP: coef. $-4,20$ e p-valor 0,14$)$.

Para Malmendier e Tate $(2005,2008)$, o acúmulo de cargos de CEO e presidente do conselho de administração (PCA) indica a existência de ECO, dado que na inexistência de outro cargo de elevada hierarquia comparável a esses, e que possa intervir em seus desvios cognitivos, indivíduos com tais características tendem a tomar decisões enviesadas. Apesar dessas suspeitas, os resultados da pesquisa não apresentaram significância para esta característica (ISF: coef. -1,88 e p-valor 0,58; ISFA e ISFP: coef. $-1,77$ e p-valor 0,60 ). Talvez esse fato possa ser pela regra de boa governança de que uma pessoa não assuma os dois cargos de forma conjunta, uma vez que uma das atribuições do PCA é supervisionar as decisões tomadas pelo CEO. Nesse sentido, os CEO's que são PCA podem estar evitando emitir sinalização de seu ECO pela compra e venda de ações, tendo em vista que o mercado deverá acompanhalo de maneira mais efetiva.

O CEO pode buscar momentos propícios para adquirir ações, o que pode estar refletido em 
indicadores como o índice P/L. Esse índice mede a qualidade do preço da ação das empresas (Kimura, 2003; Aguiar et al., 2008). Os resultados não confirmaram as suspeitas levantadas (ISF: coef. -0,01 e pvalor 0,54 ; ISFA e ISFP: coef. - 0,01 e p-valor 0,52 ), de que o CEO com comportamentos excessivamente confiantes e otimistas comprariam ações em momentos menos propícios.

Além do índice $\mathrm{P} / \mathrm{L}$, um importante parâmetro para as decisões de compra ou venda de ações é o risco da empresa (Dalmácio, Lopes, Rezende, \& Sarlo, 2013; Gatsios, 2013). A regressão, no entanto, não indicou significância na relação (ISF: coef. - 0,00 e p-valor 0,92. ISFA e ISFP: coef. - 0,00 e p-valor $0,80)$.

Existem indícios de que os investidores venham a adquirir ações principalmente em períodos de crescimento do PIB (Lélis et al., 2015), uma vez que essas pessoas tenderiam a apresentar maiores rendimentos nesses períodos e poderiam efetuar novos investimentos. Contudo, acredita-se que os CEO's excessivamente confiantes e otimistas venham a efetuar maiores compras do que vendas de ações das empresas em que atuam, mesmo em períodos subsequentes à queda da economia (PIB baixo), pois acreditam que as ações estão cotadas a um preço baixo do ideal, e que posteriormente com um bom momento da economia, atrelado à sua boa performance como gestor, as ações apresentariam aumentos consideráveis em seus valores. Os resultados de ambas as regressões não demonstraram significância estatística para essa variável (ISF: coef. -0,54 e p-valor 0,21; ISFA e ISFP: coef. -0,48 e p-valor 0,26).

Além das variáveis de controle destacadas pela literatura e apresentadas na Tabela 3, foram rodados testes de regressão com outras variáveis, tento em vista aumentar a robustez das análises. Para esses testes, considerou-se as variáveis do modelo 2 (em que se encontrou significância na variável ISFA), inserindo ainda: (1) controle do ano; (2) controle do setor; e (3) controle do setor por ano de estudo. Nos dois primeiros testes foram, as variáveis de controle adicionadas foram mensuradas por dummy, e para o último teste considerou-se a realização de uma dummy multiplicativa (setor*ano). Ao efetuar o primeiro controle (1) a variável ano não se mostrou significante, e os demais resultados da pesquisa se mantiveram. Os setores de atuação (controle 2) mostraram-se significantes no Consumo não Cíclico a um nível de 5\%, e no Financeiro e Outros e Materiais Básicos à um nível de $10 \%$, e os resultados da pesquisa se mantiveram. Assim como, se mantiveram no último teste, o qual apresentou significância ainda para a relação Consumo Não Cíclico no ano de 2012 a um nível de 5\%, e para as relações setor Materiais Básicos e Tecnologia da Informação para o ano de 2012 a um nível de 10\%.

Essa seção procurou-se testar a hipótese de teste de que a Sofisticação Financeira do CEO está relacionada positiva e significativamente ao ECO. Através do teste dos dois índices de sofisticação tomados separadamente no modelo econométrico 2, foi possível observar uma relação positiva e significativa entre o índice de sofisticação financeira acadêmica (ISFA) e os vieses de Excesso de Confiança e Otimismo ECO. Assim, a hipótese 1 é parcialmente aceita, uma vez o ISFA é positiva e significativamente relacionado ao ECO.

\section{Discussão e Conclusão}

É notória a importância de se entender os desvios cognitivos individuais em indivíduos que ocupam cargos onde lhes são exigidas decisões instantâneas frente a situações que envolvam incerteza. Mesmo que o indivíduo apresente elevada Sofisticação Financeira, ao agir intuitivamente tem afetado o seu conhecimento tácito (Paraskevas et al., 2013), responsável pelo armazenado dos conhecimentos técnicos e emoções, essencialmente necessários para tomar decisões. Tendo em vista que o cargo de gestor é frequentemente exposto a pressões, e que esse fato o leva a usar o pensamento intuitivo (sistema 1) tornando-o propenso a cometer desvios cognitivos, é importante considerar assim como Barros (2005), que possíveis desvios de racionalidade são capazes de interferir na tomada de decisões. Nesse sentido, o estudo testou a relação entre a Sofisticação Financeira dos CEO's e os vieses cognitivos Excesso de Confiança e Otimismo 
A análise econométrica aponta para uma relação positiva e significativa entre o Índice de Sofisticação Financeira Acadêmica (composto pelos itens Formação Internacional, Formação Financeira e Experiência no Ramo) e o excesso de confiança e otimismo, indicando que quanto maior for a sofisticação financeira acadêmica do CEO, maior é o excesso de confiança e otimismo. Esses resultados possibilitam a aceitação parcial da hipótese alternativa de que a Sofisticação Financeira estaria relacionada positiva e significativamente com o excesso de confiança e otimismo, pois tal relação foi detectada na dimensão acadêmica (embora o índice apresente um item relacionado à experiência). Também, foram efetuados testes de robustez do modelo considerando as variáveis setor e ano, sendo que os resultados da pesquisa se mantiveram consistentes. Ao realizar o teste com o modelo logaritmizado tornou possível a identificação da força explicativa de $15 \%$ do Índice de Sofisticação Financeira Acadêmica sobre a variável dependente. Analiticamente, é possível dizer que a Formação Internacional, Formação Financeira e Experiência no Ramo explicam respectivamente 5,53\%, 5,36\% e $4,11 \%$ do excesso de confiança e otimismo do CEO, considerando o nível de participação de cada um dos itens na construção do índice.

Os resultados encontrados nesse estudo, contribuem com a literatura existente sobre os vieses cognitivos excesso de confiança e otimismo, uma vez que foram identificadas características dos gestores que podem vir a desencadear um comportamento enviesado. Tais características se referem aos itens formação internacional, formação financeira e experiência no ramo, que compuseram a sofisticação financeira acadêmica. Destaca-se que até o momento, foi encontrado apenas o trabalho realizado pelos pesquisadores Mishra e Metilda (2015), em que houve uma tentativa de explicar o excesso de confiança e otimismo, contudo considerando o comportamento dos investidores como objeto de estudo. Entendese que pesquisas futuras podem vir a considerar as características destacadas ao estudar o comportamento dos CEO's, podendo e, inclusive, adicionar novos itens que possam vir a contribuir na identificação possíveis causas do excesso de confiança e otimismo. Por fim, após a realização do estudo, percebe-se, que há pouca compreensão do que leva os indivíduos a se tornar excessivamente confiante e otimistas, e assim ampliar a identificação e compreensão das possíveis consequências positivas e negativas que a presença desses vieses pode vir a provocar no comportamento de um indivíduo responsável por importantes decisões sobre uma empresa. Pode-se considerar que este trabalho possui limitações. A variável ECO foi mensurada por uma proxy que, descartou empresas em que não houve negociação de ações por parte da diretoria no período. Se por um lado este procedimento aumenta a possibilidade deste tipo de transação representar o excesso de confiança e o otimismo, pois garante que não haja restrições às compras ou vendas, por outro reduziu o tamanho da amostra e a restringiu a empresas com negociações reduzindo assim sua representatividade. Assim, considera-se que as possibilidades de generalização são limitadas, sendo necessário que haja estudos adicionais que busquem confirmar ou refutar os achados utilizando outras proxies, além de amostras diferenciadas. Ainda, o fato de o estudo ter sido conduzido no mercado brasileiro torna inviável a generalização para outros países, mas abre a possibilidade de testes em países com características diferentes.

\section{Referências}

Aguiar, R. A., Sales, R. M., \& Sousa, L. A. de (2008). Um modelo Fuzzy comportamental para análise de sobre-reação e sub-reação no mercado de ações brasileiro. Revista de Administração de Empresas, 48(3), 8-22. http://doi.org/10.1590/S0034-75902008000300002

Andriosopoulos, D., Andriosopoulos, K., \& Hoque, H. (2013). Information disclosure, CEO overconfidence, and share buyback completion rates. Journal of Banking \& Finance, 37(12), 5486-5499. https://doi.org/10.1016/j.jbankfin.2013.04.011

Antonczyk, R. C., \& Salzmann, A. J. (2014). Overconfidence and optimism: The effect of national culture on capital structure. Research in International Business and Finance, 31, 132-151. https://doi.org/10.1016/j.ribaf.2013.06.005 
Barros, L. A. B. D. C. (2005). Decisões de financiamento e de investimento das empresas sob a ótica de gestores otimistas e excessivamente confiantes (Tese de doutorado). Universidade de São Paulo, Faculdade de Economia, Administração e Contabilidade de São Paulo, São Paulo, SP, Brasil.

Bharati, R., Doellman, T., \& Fu, X. (2016). CEO confidence and stock returns. Jorrnal of Contemporary Accounting \& Economics, 12(1), 89-110. https://doi.org/10.1016/j.jcae.2016.02.006

Bollaert, H., \& Petit, V. (2010). Beyond the dark side of executive psychology: Current research and new directions. European Management Journal, 28(5), 362-376. https://doi.org/10.1016/j.emj.2010.01.001

[B] $]^{3}$ - Brasil, Bolsa e Balcão. (2017). Grendene SA. relatórios estruturados. Formulário de referência. 13. Remuneração dos administradores. 13.5 Remuneração baseada em ações. Recuperado em 28 de novembro, 2018, de https://www.rad.cvm.gov.br/ENETCONSULTA/frmGerenciaPaginaFRE.aspx?NumeroSequenc ialDocumento $=73886 \&$ CodigoTipoInstituica $=2$

Carr, S. C., Inkson, K., \& Thorn, K. (2005). From global careers to talent flow: Reinterpreting "brain drain”. Journal of World Business, 40(4), 386-398. https://doi.org/10.1016/j.jwb.2005.08.006

Choo, A. S., Nag, R., \& Xia, Y. (2015). The role of executive problem solving in knowledge accumulation and manufacturing improvements. Journal of Operations Management, 36(1), 6374. https://doi.org/10.1016/j.jom.2015.03.001

Custódio, C., Ferreira, M. A., \& Matos, P. (2013). Generalists versus specialists: Lifetime work experience and chief executive officer pay. Journal of Financial Economics, 108(2), 471-492. https://doi.org/10.1016/j.jfineco.2013.01.001

Dalmácio, F. Z., Lopes, A. B., Rezende, A. J., \& Sarlo, A. (2013). Uma análise da relação entre governança corporativa e acurácia das previsões dos analistas do mercado brasileiro. Revista de Administração Mackenzie, 14(5), 104. http://doi.org/10.1590/S1678-69712013000500005

Dauth, T., Pronobis, P., \& Schmid, S. (2017). Exploring the link between internationalization of top management and accounting quality: The CFO's international experience matters. International Business Review, 26(1), 71-88. https://doi.org/10.1016/j.ibusrev.2016.05.007

Davis, S. A., DeBode, J. D., \& Ketchen, D. J., Jr. (2013). Dollars and sense: The implications of CEO compensation for organizational performance. Business Hor, 56(5), 537-542. https://doi.org/10.1016/j.bushor.2013.05.008

Dedman, E., \& Lin, S. W.-J. (2002). Shareholder wealth effects of CEO departures: Evidence from the UK. Journal of Corporate Finance, 8(1), 81-104. https://doi.org/10.1016/S0929-1199(01)00027$\mathrm{X}$

Deshmukh, S., Goel, A. M., \& Howe, K. M. (2013). CEO overconfidence and dividend policy. J. Finan. Intermediation, 22(3), 440-463. https://doi.org/10.1016/j.jfi.2013.02.003

Dickel, D., \& Moura, G. L. (2016). Organizational performance evaluation in intangible: A model based on knowledge management and innovation management. Revista de Administração e Inovação, 13(3), 211-220. https://doi.org/10.1016/j.rai.2016.06.005

Faulconbridge, J. R., Beaverstock, J. V., Hall, S., \& Hewitson, A. (2009). The 'war for talente': The gatekeeper role of executive search firms in elite labour markets. Geoforum, 40(5), 800-808. https://doi.org/10.1016/j.geoforum.2009.02.001

Fellner, G., \& Krügel, S. (2012). Judgmental overconfidence: Three measures, one bias? Journal of Economic Psychology, 33(1), 142-154. https://doi.org/10.1016/j.joep.2011.07.008 
Field, A. (2009). Descobrindo a estatística usando o SPSS (2a ed.). Porto Alegre: Artmed.

Gatsios, R. C. (2013). Acurácia e dispersão das estimativas dos analistas no mercado de capitais brasileiro: Impacto da adoção do padrão IFRS sobre a qualidade preditiva da informação contábil (Dissertação de mestrado), Universidade de São Paulo, Faculdade de Economia, Administração e Contabilidade de Ribeirão Preto, Ribeirão Preto SP, Brasil.

Gervais, S., Hearton, J. B., \& Odean, T. (2003). Overconfidence, investment policy, and executive stock options. Rodney L. White Center for Financial Research Working Paper. Recuperado em 31 de outubro, 2016

de https://pdfs.semanticscholar.org/79ba/4e8749693e0d078b503751984a4dd4737006.pdf

Grip, A. D., \& Sauermann, J. (2013). The effect of training on productivity: The transfer of on-the-job training from the perspective of economics. Educational Research Review, 8, 28-36. https://doi.org/10.1016/j.edurev.2012.05.005

Hackbarth, D. (2008). Managerial traits and capital structure decisions. Journal of Financial and Quantitative Analysis, 43(4), 843-882. https://doi.org/10.1017/S002210900001437X

Hackbarth, D. (2009). Determinants of corporate borrowing: A behavioral perspective. Journal of Corporate Finance, 15(4), 389-411. https://doi.org/10.1016/j.jcorpfin.2009.02.001

Hill, A. D., Kern, D. A., \& White, M. A. (2014). Are we overconfident in executive overconfidence research? An examination of the convergence and contented validity of extant unobtrusive measures. Journal of Business Research, 67(7), 1414-1420. https://doi.org/10.1016/j.jbusres.2013.08.011

Hirshleifer, D., Low, A., \& Teoh, S. H. (2012). Are overconfident CEOs better innovators? The Journal of Finance, 67(4), 1457-1498. https://doi.org/10.1111/j.1540-6261.2012.01753.x

Huang, J., \& Kisgen, D. J. (2013). Gender and corporate finance: Are male executives overconfident relative to female executives? Journal of Financial Economics, 108(3), 822-839. https://doi.org/10.1016/j.jfineco.2012.12.005

Huang, R., Tan, K. J. K., \& Faff, R. W. (2016). CEO overconfidence and corporate debt maturity. Journal of Corporate Finance, 36, 93-110. https://doi.org/10.1016/j.jcorpfin.2015.10.009

Jemaiel, S., Mamoghli, C., \& Seddiki, M. W. (2013). An experimental analysis of over-confidence. American Journal of Industrial and Business Management, 3(4), 395-417. https://doi.org/10.4236/ajibm.2013.34047

Kahneman, D. (2003). A perspective on judgment and choice: Mapping bounded rationality. American Psychologist, 58(9), 697-720. https://doi.org/10.1037/0003-066X.58.9.697

Kahneman, D. (2012). Rápido e devagar: Duas formas de pensar (C. de A. Leite, Trad.). Rio de Janeiro: Objetiva.

Kausel, E. E., Culbertson, S. S., \& Madrid, H. P. (2016). Overconfidence in personnel selection: When and why unstructured interview information can hurt hiring decisions. Organizational Behavior and Human Decision Processes, 137, 27-44. https://doi.org/10.1016/j.obhdp.2016.07.005

Kimura, H. (2003). Aspectos comportamentais associados às reações do mercado de capitais. Revista de Administração de Empresa, 2(1), 2-14. http://doi.org/10.1590/S1676-56482003000100007

Lambert, J., Bessière, V., \& N'Goala, G. (2012). Does expertise influence the impact of overconfidence on judgment, valuation and investment decision? Journal of Economic Psychology, 33(6), 11151128. https://doi.org/10.1016/j.joep.2012.07.007 
Lélis, M. T. C., Bredow, S. M. S., \& Cunha, A. M. (2015). Determinantes macroeconômicos dos investimentos no Brasil: Um estudo para o período de 1996 - 2012. Revista de Economia Contemporânea, 19(2), 203-234. http://doi.org/10.1590/198055271922

Li, C., Sun, L., \& Ettredge, M. (2010). Financial executive qualifications, financial executive turnover, and adverse SOX 404 opinions. Journal of Accounting and Economics, 50(1), 93-110. https://doi.org/10.1016/j.jacceco.2010.01.003

Li, J., Wei, M., \& Lin, B. (2016). Does top executives' US experience matter? Evidence from US-listed Chinese firms. China Jornal of Accounting Research, 9(4), 267-282. https://doi.org/10.1016/j.cjar.2015.11.002

Lin, W.- T., \& Liu, Y. (2012). Successor characteristics, organizational slack, and change in the degree of firm internationalisation. International Business Review, 21(1), 89-101. https://doi.org/10.1016/j.ibusrev.2011.01.001

Malmendier, U., \& Tate, G. (2005). CEO overconfidence and corporate investment. The Journal of Finance, 60(6), 2661-2700. https://doi.org/10.3386/w10807

Malmendier, U., \& Tate, G. (2008). Who makes acquisitions? CEO overconfidence and the market's reaction. Journal of Financial Economics, 89(1), 20-43. https://doi.org/10.1016/j.jfineco.2007.07.002

Manfredi, S. M. (1998). Trabalho, qualificação e competência profissional - das dimensões conceituais e políticas. Educação \& Sociedade, 19(64), 13-49. http://doi.org/10.1590/S010173301998000300002

Mishra, K. C., \& Metilda, M. J. (2015). A study on the impact of investment experience, gender, and level of education on overconfidence and self-atribution bias. IIMB Management Review, 27(4), 228-239. https://doi.org/10.1016/j.iimb.2015.09.001

Oliva, E. D. C., \& Albuquerque, L. G. D. (2007). Conselheiros, CEO’s e diretores: Há alinhamento entre nomeação, sucessão e avaliação com o sistema de remuneração praticado? Revista Alcance, 12(2), 247-266. http://doi.org/10.14210/alcance.v14n2.p247-266

Paraskevas, A., Altinay, L., McLean, J., \& Cooper, C. (2013). Crisis knowledge in tourism: Types, flows and governance. Annals of Tourism Research, 41, 130-152. https://doi.org/10.1016/j.annals.2012.12.005

Paton, R. A.; \& Wagner, R. (2014). Management Education Makes a Difference: Enhancing German Engineering Performance. Long Range Planning, 47(5), 277-298. https://doi.org/10.1016/j.lrp.2012.06.002

Rezende, Y. (2002). Informação para negócios: Os novos agentes do conhecimento e a gestão do capital intelectual. Ciência da Informação, 31(1), 75-83. http://doi.org/10.1590/S010019652002000100008

Saxena, P., \& Bendale, S. (2014). The management education (MBA) Challenge a study of managerial competency needs \& how well MBA's differentiate. Procedia Economics and Finance, 11, 642653. https://doi.org/10.1016/S2212-5671(14)00230-5

Torres, P., \& Augusto, M. (2016). The impacto of experiential learning on managers' strategic competencies and style. Journal of Innovation \& Knowledge, 2(1), 10-14. Recuperado em 31 de outubro, 2016 de http://www.sciencedirect.com/science/article/pii/S2444569X1630004X. https://doi.org/10.1016/j.jik.2016.06.001 
Trejo, J. M., Gutiérrez, J. S., \& Guzman, G. M. (2016). The customer knowledge management and innovation. Contaduría y Administración, 61(3), 456-477. https://doi.org/10.1016/j.cya.2015.11.011

Yim, S. (2013). The acquisitiveness of youth: CEO age and acquisition behavior. Journal of Financial Economics, 108(1), 250-273. https://doi.org/10.1016/j.jfineco.2012.11.003

Wang, Y., Chen, C. R., Chen, L., \& Huang, Y. S. (2016). Overinvestment, inflation uncertainty, and managerial overconfidence: Firm level analysis of Chinese corporations. North American Journal of Economics and Finance, 38, 54-69. https://doi.org/10.1016/j.najef.2016.07.001

Wooldridge, J. M. (2005). Introductory econometrics. A modern approach (2nd ed.). Mason, OH: Thomson South-Western.

\author{
Autores \\ Cassiana Bortoli \\ Rua Prefeito Lothario Meissner, 632, Jardim Botânico, 82590-300, Curitiba, PR, Brasil \\ E-mail: cassianabortoli@gmail.com \\ Rodrigo Oliveira Soares \\ Rua Prefeito Lothario Meissner, 632, Jardim Botânico, 82590-300, Curitiba, PR, Brasil \\ E-mail: rosoares@ufpr.br
}

\title{
Contribuições
}

$1^{\text {o }}$ autor: Definição do problema de pesquisa, desenvolvimento de hipóteses, fundamentação teórica, definição de procedimentos metodológicos, coleta de dados, análise estatística, análise e interpretação dos dados, redação e revisão crítica do manuscrito.

$2^{\circ}$ autor: Definição do problema de pesquisa, desenvolvimento de hipóteses, definição de procedimentos metodológicos, análise e interpretação dos dados, redação e revisão crítica do manuscrito.

\section{Financiamento}

O presente trabalho foi realizado com o apoio da Coordenação de Aperfeiçoamento de Pessoal Nível Superior (CAPES) - Código de Financiamento 001.

\section{Conflito de Interesses}

Os autores informaram que não existe conflito de interesses.

\section{Verificação de Plágio}

A RAC mantém a prática de submeter todos os documentos aprovados para publicação à verificação de plágio, mediante o emprego de ferramentas específicas, e.g.: iThenticate.

\section{Material Suplementar}

Os autores em comum acordo optaram pela não divulgação da base de dados, uma vez que há pesquisas de tese/dissertação relacionadas à continuação do tema em andamento. Essa pesquisa advém de uma Dissertação de Mestrado, que contou com apoio financeiro de uma bolsa CAPES. 